\title{
Deep Weber Dominant Local Order Based Feature Generator and Improved Convolution Neural Network for Brain Tumor Segmentation in MR Images
}

\author{
Nisha Joseph, D. Murugan, Basil John Thomas, Ramya A
}

\begin{abstract}
This paper introduces a scheme for retrieving deep features to carry out the procedure of recognising brain tumors from MR image. Initially, the MR brain image is denoised through the Modified Decision Based Unsymmetric Trimmed Median Filter (MDBUTMF) after that the contrast of the image is improved through Contrast Limited Adaptive Histogram Equalization (CLAHE). Once the pre-processing task is completed, the next phase is to extract the feature. In order to acquire the features of pre-processed images, this article offers a feature extraction technique named Deep Weber Dominant Local Order Based Feature Generator (DWDLOBFG). Once the deep features are retrieved, the next stage is to separate the brain tumor. Improved Convolution Neural Network (ICNN) is used to achieve this procedure. To explore the efficiency of deep feature extraction and in-depth machine learning methods, four performance indicators were used: Sensitivity (SEN), Jaccard Index (JI), Dice Similarity Coefficient (DSC) and Positive Predictive Value (PPV). The investigational outputs illustrated that the DWDLOBFG and ICNN achieve best outputs than existing techniques.
\end{abstract}

Keywords: MR Brain Tumor, CLAHE, Machine Learning Scheme, MDBUTMF, DWDLOBFG and ICNN.

\section{INTRODUCTION}

Without an automated tool to help with a doctor's diagnosis and a limited amount of medications, doctors can use not only high risk of misdiagnosis, but also increase patient wait times. Doctors should take the time to review the results of the tests and pictures by hand instead of spending time with the patient. In order to improve patient care, advanced medical technology is needed in the form of tools to increase the efficiency of the physician and to decrease patient tolerance during hospitalization and recovery. The

Revised Manuscript Received on February 25, 2020.

* Correspondence Author

Nisha Joseph*, Research Scholar, Dept. of Computer Science and Engineering, Manonmaniam Sundaranar University, Abishekapatti, Tirunelveli-627012, Tamilnadu, India. E-mail: nishajoseph226@gmail.com

D. Murugan, Prof \& Head, Dept. of Computer Science and Engineering, Manonmaniam Sundaranar University, Tirunelveli, Tamilnadu, India. E-mail: dhanushkodim@yahoo.com

Basil John Thomas, Assistant Professor, Dept. of Business Administration, Sur University College, Sultanate of Oman. E-mail: drbasiljt@gmail.com

Ramya A, Assistant Professor, B.S Abdur Rahman Crescent Institute of Science and Technology, Chennai, India. E-mail: ramyaanandan10@gmail.com

(C) The Authors. Published by Blue Eyes Intelligence Engineering and Sciences Publication (BEIESP). This is an open access article under the CC BY-NC-ND license (http://creativecommons.org/licenses/by-nc-nd/4.0/) aspiration of this study is to create an automated scheme to help doctors diagnose to prevent misdiagnosis and reduce the patient's waiting time. In particular, the study achieved this automation by classifying the brain tumor from the image of the patient's brain. The picture requires a physician to review some images of the image to determine the health problems that need time from a modern diagnosis. Our aspiration is to discover the kind of brain tumor secretly to reduce the burden of physicians, leaving the most complex symptoms.

Brain tumor is a severe ailment, where abnormal growths in the brain may interfere with the function of the brain. The NBTF statement that the quantity of people in urbanized countries with brain tumors had augmented by 300 percent more than the past three decades. Manual judgment of a brain tumor by a physician is not accurate but at all [18, 34]. Magnetic magnetism is a modern medical imaging technique that provides a wealth of information on human gestural anatomy [35]. The automatic recognition of magnetic stenosis (MRIs) has a normal and abnormal MRI target based on the absence or presence of tumors. Therefore, diagnosis can be a challenge for image classification. The widespread brain tumor has led to large MRI data. Thus, the development of the immune system for tumor diagnosis and localization is important. A deeper and more comprehensive training focuses on playing an important role in brain sculpting, division, recording and classification of tumor tissue [20,16].

In-depth study of recent studies is an important topic for research. Modules for advanced training include several layers that can be trained through a training program that manages or controls [17, 32]. In current learning, deep study models have attained noticeable outputs matched up to the ancient brain tumors and tumor photography [31, 21]. In addition, in-depth training courses such as CNN have enhanced the area of recognition and classification in many programs [33, 30]. As a model of deep learning, CNNs were employed to retrieve high quality data from raw information [24].

Even though many learning have focused on using a deep training method to diagnosing brain tumors, there is still no entire system for automatic tumor recognition and localization in literature. Additionally, integrating tumor recognition and localization in a solitary diagnostic system remains a challenge. The stage of brain tumor recognition before the localization of the tumor in a solitary system guides to the abandonment of a normal image of the process at the stage of localization. This will open the door for the introduction of autoimmune recognition systems that can save time and power from the location of the tumor in the image. 
A summary of the paper is as follows. A short literary work explains in Section 2. Section 3 presents the full technical scheme of the proposed work. Section 4 provides the datasets employed to evaluate the results and the indicators used for this evaluation. Finally, the conclusion of this work is presented in Section 5.

\section{RELATED WORK}

One of the tasks for controlling the initial energy the patient's diagnosis depends on the patient's evaluation of the patient and the results of his or her tests. The discovery of brain tumors has undergone substantial research and numerous discoveries have been demonstrated larger than the earlier period of two decades. In [1] the grouping of morphological segments, separate waveguides (DWT), PCA and KSVM were performed to classify MPT normally and abnormally. Further improvements were made to classify irregular images, such as cancer or cancer, by using two types of agents, Abd-Ellah et al. [2]. Devasena and Hemalatha [22] suggested a CAD structure for detecting abnormalities in MRIs using the HADA algorithm. In [27], which is used Patil and Udupi preprocessing, the distribution divides the features and methods of the probabilistic nerve network (PNN) for the identification of the brain tumor. Arakeri and Reddy were administered three different classes: SVM, the neurological artificial network (KNN) and the K-NN (K-NN), in order to distinguish between the type Different meat. Dandil et al. [9] Propose a system for the classification of brain tumors, where the C-Space Blankness (FCM) is applied to the division of the part of the brain tumor, and SVM is used in steps Of this assortment. Goswami and Bhaya [13] showed a new classification of brain tumors for MRT based on ANN. Binding histogram alignment, distortion filters and edge recognition were performed in the early stages. This characteristic comes from an independent component Examination (ICA).

The Self-Neural Network of Self-Neural Networks (SOMNN) is carried out in a fragmentation phase. Deepa and Devi [10] projected a system that extracted the classification and portions of the tumor. The best texture feature was taken from the image tested using statistical features. During each of the classification and separation stages, a Neural Interface (BPN) and Radial Basic Function (RBFN) are used. In the field of modernization, deeper approaches for brain tumor recognition have been found in this area. [8] It is a CT of the brain based on the deep nervous system (DNN). The Gray-Level Control Grade (GLCM) matrix is used to derive features and DNN is used for assortment. The mean accuracy of the this scheme was $83 \%$. Gao et al. [12] Recommended brain ratings included CTN 2D and 3D. The accuracy of the average distribution was $87.6 \%$ and every layer consisted of seven layers. Yan $\mathrm{Xu}$ et al. Suggested one method with profound alteration for the division and separation of the brain tumor. The accuracy of the distribution was 97.5\% [19]. Numerous methods of deep learning for the muscular buildup of the brain are offered in the literature. Havaei et al. [30] Automatically revealed the brain's muscular discovery using CNNs based DNNs with a DICE 0.88 average score. In [6] the Proper Waste Nets (FCR-NN) network was developed in combination with a fully-integrated architecture with increased waste identification. The network has 22 sophisticated layers and the resulting DICE score is 0.87 .
El-Dahshan et al. [11] used 101 images and FFBPNN method is employed to achieve 99.00 accuracy.

Devasena and Hemalatha [22] used 250 images and HADA method is employed to achieve 98.8 accuracy. Arakeri and Reddy [3] used 550 images and SVM, ANN and K-NN methods are employed to achieve 99.09 accuracy. Dandel et al. [23] used 376 images and KSVM method is employed to achieve 91.49 accuracy. Goswami and Bhaiya [13] used 70 images and Zones method is employed to achieve 98.60 accuracy. Deepa and Devi [25] used 42 images and BPN and RBFN methods are employed to achieve 85.71 accuracy. Abd-Ellah et al. [18] used 80 images and KSVM method is employed to achieve 100 accuracy. Adult - Elul and El. [18] used 120 images and KSVM method is employed to achieve 100 accuracy [5] Requested 3D CPU usage for parts of the abnormal part of the MRI, based on a network that uses many features and has DICE 0.84 scores. Zhao et al. [36] Utilized the Convolutional Neural Network (FCNN) and the CRF, a brain-based therapy with DICE 0.87. Pereira et al. [28] is a preliminary Examination of the NMR-based FCN-based FCN-based muscle paralysis, a preliminary treatment step that demonstrates the DICE score of 0.85 .

Recently, Pereira et al. [29] Explore full seamless system that relates to $\mathrm{CNN}$ recycling with post-processing. The recent system can attain a DICE score of 0.88 in the cost of the difficulty of the system. Jun Cheng et al. [7] has created a two-stage tumor-based method to creating offline databases and online downloads. In the non-parabolic system, the image of the brain tumor is processed step by step. These steps include tumor segment retrieving and distance learning. In online learning, the brain input will be treated in a similar way, and compares the functions extracted with the distance measurements learned in the online database. This method does not use neural networking methods, but can attain an assortment of $94,68 \%$. Conversely, Gawande along with Mendre employ a deep nervous system using an automatic gastroenterologist. Image splitting and download features are performed on the image before being processed by the DNN layer. Textures based on texture and intensity are extracted using the GLCM matrix and the Dynamic Circle Movement (DWT).

In the final step, the DNN layer is made, with two autocorrels and a layer of softmax for classification. In addition, Pereira et al. [4] Also learn how to use c. N. Yes. With 3x3 small forces to reach deep architecture and stay away from reorganization. They also explore the normal use of intensity as a healing stage previous to entering CNN's layers. In this learning, enthused by these developments, we thoroughly examine and investigate the CNN in the assortment of many brain tumors to get more accurate.

To conquer the above boundaries of earlier brain tumor recognition structures, this work suggest an process rooted in hybrid features that comprise data from useful as well as deep training options. The method we suggest is in four ways.

- First, we know that this is the earliest method for the MR Recognition System, using a combination of hand-drawn and deep images. By combining deep and artificial images, we enhance the accuracy of recognition matched up to to the most advanced discovery schemes and decrease the recognition differences in the effects of the MR images.

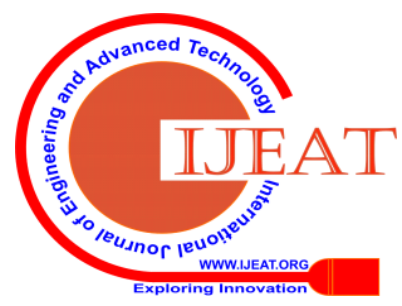


- Secondly, instead of using some of the pre-prepared ICNN templates to retrieve image features from earlier learning $[34,35]$, we can get a single ICNN model employing larger images and preview images to download. Features. By employing this method, we create the CNN system more appropriate for the brain tumor recognition system's recognition system along with decrease the complexity of recognition systems matched up to earlier research.

- Third, we employ two approaches to combine the recognizing outputs obtained by deep-angled features along with hands that include leveling and functional level synthesis. For synthesis, weight-loss results were obtained to combine the functions of the picture, which were drawn by hand, to clearly illustrate the characters of the brain tumor difficulty for the MR Recognition System..

- At last, through [36] we have prepared our trained ICNN model with all the PAD techniques enabled other researchers to compare them with our method..

In the next segment, this work introduce a method for identifying and classifying brain tumors that diminish by defeating the boundaries inherent in this problem.

\section{MATERIALS AND METHODS}

The general architecture of brain tumors separation based on supervised learning is presented in Fig. 1. Initially, brain MR images were denoised by using MDBUTMF moreover the image contrast is improved through CLAHE. Once the pre-processing task is completed, the next action is to extract the feature from the denoised and contrast improved image.

To extract features from pre-processed images, the work provides a feature-extraction technique called Multi Transform Encoded Deep Zig Zag Local Binary Pattern (MTEDZZLBP). After extracting these deep features, the next action is to separate the brain tumor. Supervised brain separating method is used to perform this process. Among the supervised schemes, this work uses an in-depth learning method called CNN. Finally, the output function is provided as input to these machines knowledge schemes to separate the brain tumor areas.

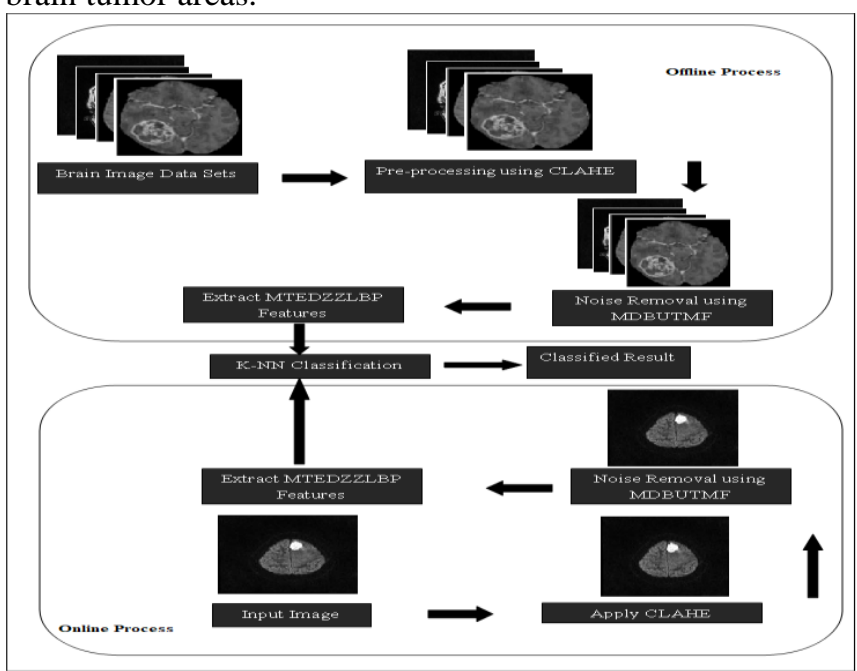

\section{A. Pre-Processing}

Initially, input MR brain was improved through Adaptive Histogram Equalization (AHE) technique. AHE is a computerized image processing technology used to enhance
Fig.1. Complete Design of the Proposed Method

image contrast levels. Contrast-enhanced MR images and conventional MR images are shown in Fig. 2.
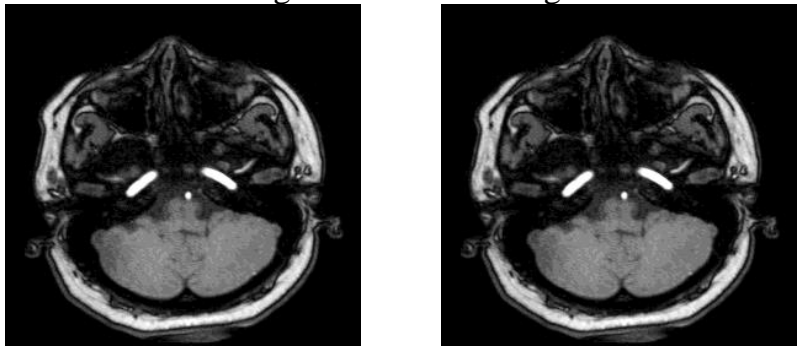

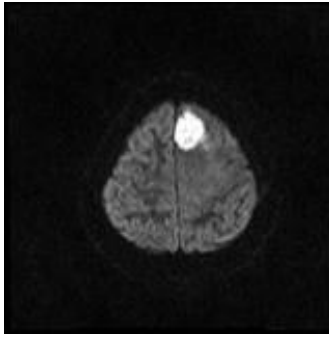

(a)

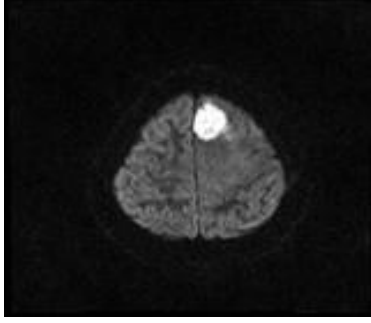

(b)
Fig.2(a). Input MRI 2(b). Contrast Improved MRI

Then Modified Decision Based Unsymmetric Trimmed Median Filter (MDBUTMF) was used for de-noising. Here we follow the algorithm of anisotropic diffusion with tukey edge stopping function. Basically in AD scheme, a good edge-preserving behavior is depends upon the selection of right conductance function. Kamalaveni et al [8] suggested that the tukey edge recognition function is extremely proficient in protecting sharp edges, fine details as well as visual quality of the reconstruct image is base to be true. But edge stopping function is unable of denoising efficiently. To overcome this drawback, Gaussian filter is employed to filter out the noise. Where is a Gaussian filter of scale. This means that the local gradients that are the parameter of edge stopping function are also calculated using a smoothed version of the image in every iteration. The denoised MR images with MDBUTMF are illustrated in Fig.3.
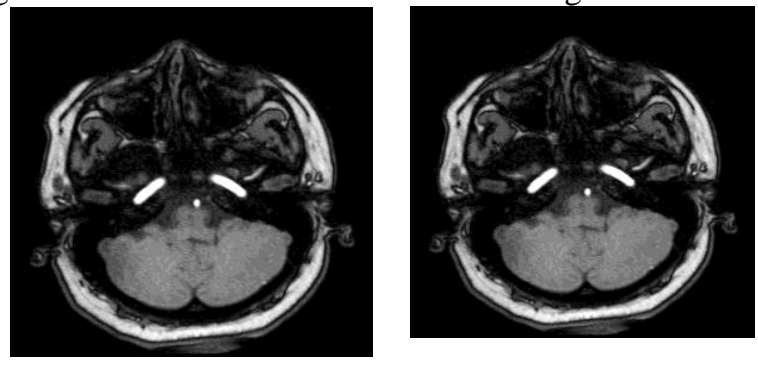

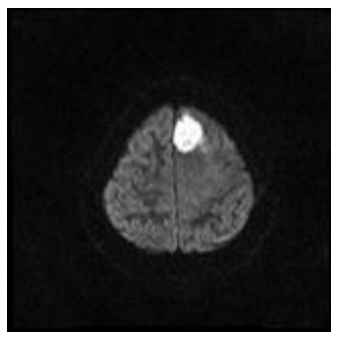

(a)

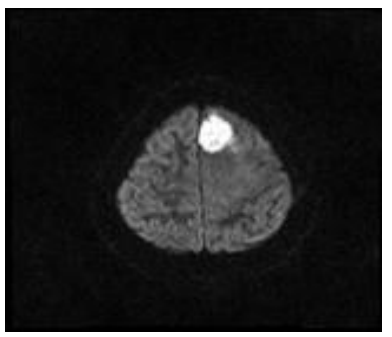

(b)
Fig.3(a). Histogram Normalized image, 3(b). MDBUTMF Filtered image 


\section{B. Feature Retrieval}

Following the procedure of denoising along with contrast improvement, the later procedure is to extract the features from the image of the brain. This work created an innovative concept for extracting deep features from an MR image called Deep Weber Dominant Local Order Based Feature Generator (DWDLOBFG).

\section{Local Order-Based Feature Generation (LOBFG)}

In this section, talks about method called local order based feature generation method (LOBFG). The human brain system is perceptive to local modification, on the contrary to the complete dimensions of the image representing the texture description.

This features are computed by five steps.

1. Find Gradient Response of Image

2. Apply Mean Filter on Gradient Response of Image

3. Find the Neighbourhood data

4. Find the Order Sequence of the Neighbourhood Data

5. Find Description value based on Codebook.

\section{Find Gradient Response of Image}

In this section first get the input image $\mathrm{S}$ Then assuming the $\mathrm{x}$ - and $\mathrm{y}$-axis gradient responses of image I by using the equation (1) and (2).

$G R I_{x}=-\frac{1}{2} \cdot S(x-1, y)+0 . S(x, y)+\frac{1}{2} \cdot S(x+1, y)$
$G R I_{y}=-\frac{1}{2} \cdot S(x, y-1)+0 . S(x, y)+\frac{1}{2} \cdot S(x, y+1)$

where $G R I_{x}$ is the gradient response image of $\mathrm{x}$ axis and $G R I_{y}$ is the gradient response image of y axis. $S(x, y)$ is the input image. This is shown in Figure.4.
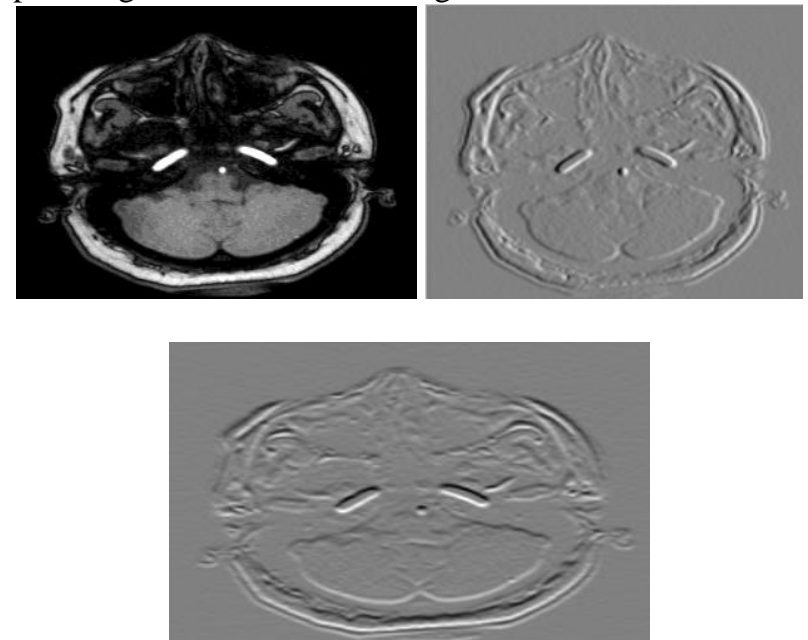

Fig.4 Gradient Response Image

Apply Mean Filter on Gradient Response of Image

In this section, the $3 \times 3$ size mean filter is applied on gradient magnitude of Image which is get by the previous step. This is shown in Figure.5.
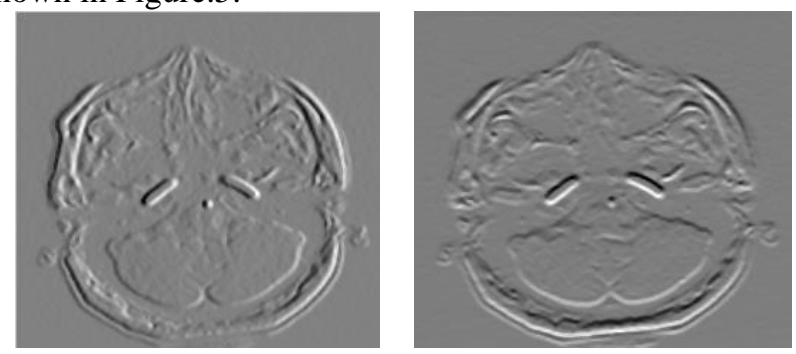

Fig.5 Median Filtered Gradient Response Image

\section{Find the Neighbourhood data}

In this stage, for any pixel its four neighbourhoods vector is found and captioned with (1, 2, 3, and 4) in the clockwise direction. Let take 3x3 mean filtered gradient sample image as show in Figure.6. to see the detailed explain.

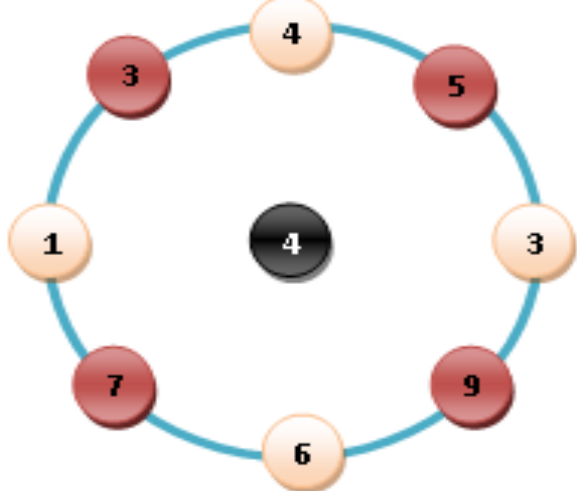

Fig. 6 3x3 Median Filtered Gradient Sample Image In the above image the center pixel value is 45 . Now the four neighbourhood pixels of 45 is taken. For this follow the anyone pattern which is show in Figure.7.
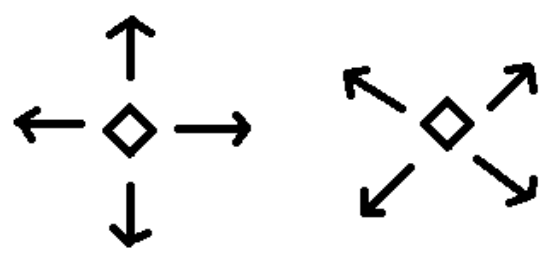

Fig. 7 Neighbour Pattern Image

This project follow the first pattern. Using this pattern the four neighbourhood data of the center pixel 45 is $(47,30,64,11)$. And label these data as $1,2,3,4$. This is show in Figure.8.

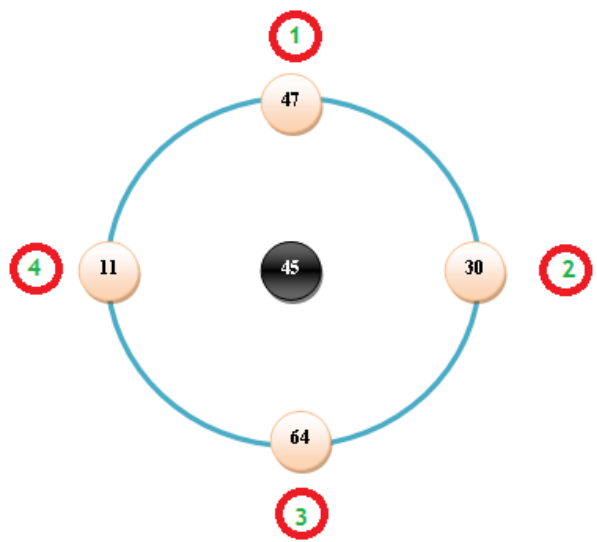

Fig. 8 Neighbour Pattern Applied on Image

Find the Order Sequence of the Neighbourhood Data

After finding the neighbourhood data then these four neighbors are sorted as index in ascending order. So the sorted index values 4,2,1,3 are shown in Figure.9. 


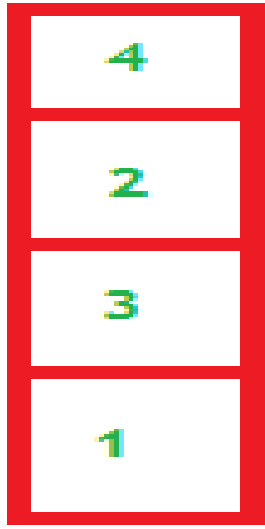

Fig. 9 Sorted Index Value

Find Description value based on Codebook

Finally, find and extract an index vector from a code book. This method shows that its position in the codecs is thirty-one, e.g. So thirty-one, so this method encodes the position of the star as 31. This is illustrated in Figure 10.

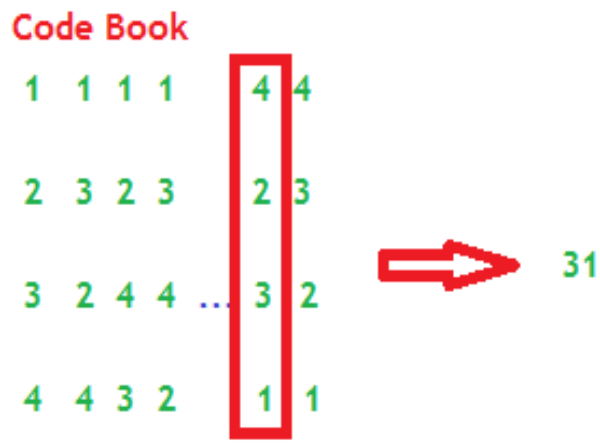

Fig.10 Find Decryption from Codebook

The algorithm and flowchart of this method is given below in very detailed manner.

Algorithm of LOBFG

Input : Image $\mathrm{S}$, Present Pixel value $S_{c}$, Present $\mathrm{X}$ Index, Present Y Index, Pixel Distance $P$, Whole Number of Neighbours T

Output: LOBFG Array

Algorithm :

Follow the steps below for all pixels in the input image

1. Acquire the neighboring pixels Sp of present pixel Sc based on the pixel Distance P.

2. Apply gradient response of $\mathrm{x}$ axis and $\mathrm{y}$ axis on $\mathrm{Sp}$ and Sc to exchange pixel into gradient value GV.

3. And then apply mean filter on to the gradient value GV.

4. Then take the neighbourhood data of the current mean filtered GV

5. And then sort these neighbourhood value in ascending order.

6. After that find the sorting index value and compare it into the code book to find the LOBFG description value

7. This is named LOBFG values

8. After that stock up the values into the array. This array is named bin

Deep Weber Dominant Local Order Based Feature Generator (DWDLOBFG)
This section proposes a method for retrieving features from MR input images. This method is called Deep Weber Dominant Local Order Based Feature Generator. Although WDLOBF produce best result than the past feature retrieving schemes, adding together deep learning with WDLOBF surely increase the recognition accuracy. Because, deep learning using the deep features by increasing the size of the features. Generally supervised classification result is based on the no of training samples and the total no of features are given to it. If the number of features are increased the classifier produce best result. So in this section to enhance the recognition accuracy of the WDLOBF the deep features are summed to the WDLOBF. To retrieve the deep feature CNN is used. The CNN is applied after retrieving the WDLOBF feature map from the input MR image. Therefore, in this work, the $\mathrm{CNN}$ is formed through three convolutional layers with pooling layers.

\section{Convolution Layer}

In a CNN, convolution layers is one of the main circuits for functional extraction. At the edge of this paper, the gradient and angle characteristics are taken out from the convolution layers. Usually MR image includes coarse along with fine data. Therefore, this work employs edge, corner as well as gradient techniques to exploit the fine features of MR imaging. To extract first, a filter is created and then perform a compression operation using the following formula to derive the function.

$$
\begin{aligned}
& F I(i, j)= \\
& \sum_{m=-1}^{1} \sum_{n=-1}^{1} F M I\left(i+m_{x} j+n\right) \times K(m, n)
\end{aligned}
$$

where $F I(i, j)$ is the convolution result of the GOLOF feature map with FMI. Here $i$ and $j$ are variables to symbolize the location of image. $K$ is the convolution filter of size $m \times n$.

\section{Pooling Layer}

This layer of assembly must apply a lower model, ie. Reduce calculation time by dropping the extraction characteristics.

There are two types of inlay layers: maximum combination and average input. Max. Input, we enter only the largest pixel value of all pixels in the filter field. In the case of a merger we average, we take the mean of the entire values. The resulting layered result is provided as the input of the next folding layer. After the completion of the three layers, the results of the three integral layers are joined in the form of yield. These features are labeled deep features. In the end, these features are provided in the NSN for Men's Notifications.

\section{Classification}

After extracting the function of features, the next procedure is to separate the brain image. Among the different methods of monitoring separation, this work focuses on machine learning methods. Among other methods of machine learning, this work requires an in-depth classifier called Convolution Neural Network.

\section{Improved Convolution Neural Network}

The distribution of brain tumors in the $\mathrm{CNN}$ is separated into two stages, both offline as well as online.

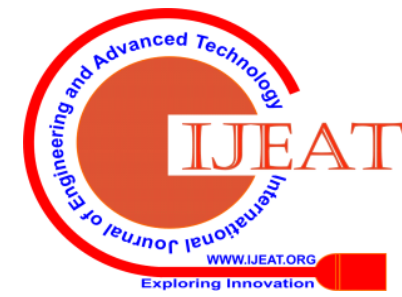


A flow chart for the recognition of brain tumors based on CNN is shown in Fig.11, the total quantity of images is alienated into dissimilar groups with label names for instance brain tumors as well as non-tumor images. etc. Pre-treatment process, extraction, and classification were performed during the training phase to produce predictive models. First, label the training image. Finally, a resolution neural network is employed to robotically categorize brain tumors. The network have to train the entire layer to the final to train the first layer. So time utilization is extremely high. This will diminish performance. To keep away from this difficulty, a model based on brain training was employed. In the proposed CNN, last layer is only trained in matlab execution.

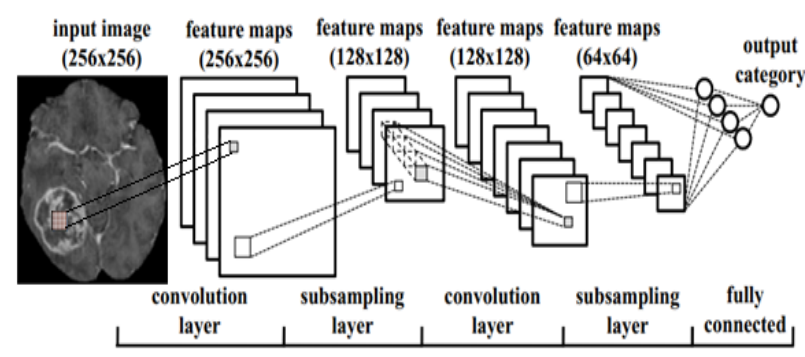

Fig. 11 Brain Tumor Recognition using ICNN

The discovered brain tumor areas by CNN is exposed in Fig.12

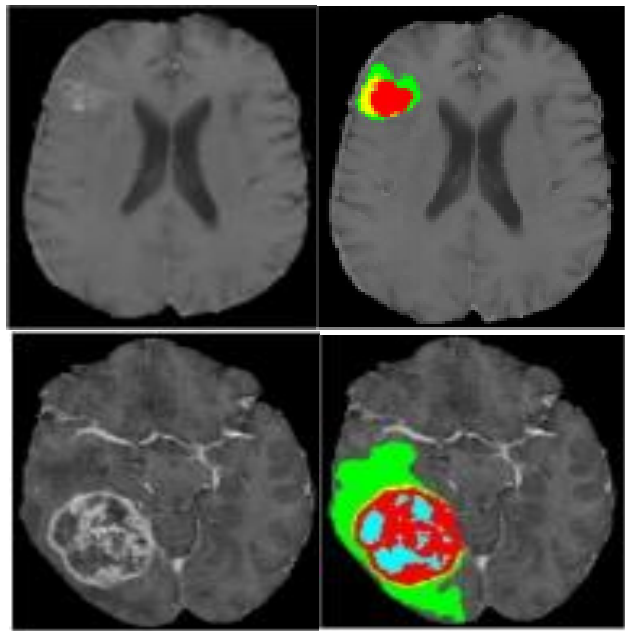

(a)

(b)

Fig.12 (a). Input MR Image, 3(b). Segmented Image by CNN

\section{RESULT AND EXAMINATION}

\section{A. Dataset Used}

The machine learning process accessible in this article was assessed on the BRATS 2015 databases. [24], [25]. These dataset are downloaded using https://www.smir.ch/BRATS/Start2015. 4,800 brain tumor images were evaluated in this work. The proposed scheme executes to images of a tumor in the brain. A 4,000-mm brain tumor was used during the training, but each had 1,000 brain tumors. It has five types of tumors, and each image has 200 images displayed. Experiments are carried out on a BRATS2015 FLAIR, T1, T1c and T2 training ground truth database of $4000 \mathrm{MR}$ images of brain tumor and 800 testing ground truth database. Figure. 13 describes the model MR images in the dataset.
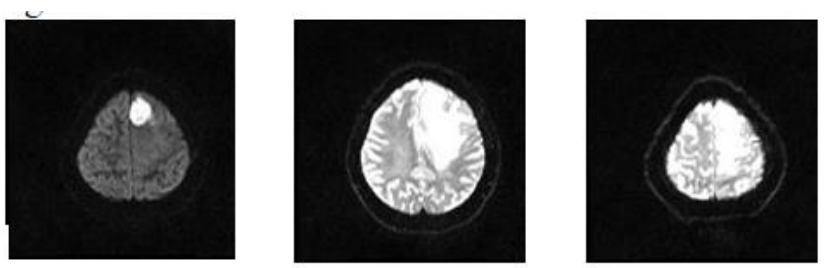

Fig.13 Experimental Images

\section{B. Evaluation Metric}

Evaluation of the brain tumor separating methods, this work uses four indicators: Sensitivity (SEN), Jaccard Index (JI), Dice Similarity Coefficient (DSC) and Positive Predictive Value (PPV). The DSC [26] metric computes overlap between basic facts as well as tumor separating methods to split results through equation (4).,

$$
\mathrm{DSC}=\frac{2 \mathrm{TP}}{\mathrm{FP}+2 \mathrm{TP}+\mathrm{FN}}
$$

PPV computes of the full amount of FP and TP through Eq.(5),

$$
\mathrm{PPV}=\frac{\mathrm{TP}}{\mathrm{FP}+\mathrm{FP}}
$$

The Jacquard index is computed as the amount of focal pixels of brain tumors, and not brain tumors, divided by the amount of their grouping through equation (6).

$$
\mathrm{JI}=\frac{\mathrm{TP}}{\mathrm{TP}+\mathrm{FP}+\mathrm{FN}}
$$

Finally, sensitivity index was computed to assess TP and FN volumes through equation (7)

$$
\mathrm{SEN}=\frac{\mathrm{TP}}{\mathrm{TP}+\mathrm{FN}}
$$

\section{Experimental Examination}

Trial No 1: Examination of Feature Retrieval Schemes

In this trial , this work will assess the proposed feature retrieval schemes.

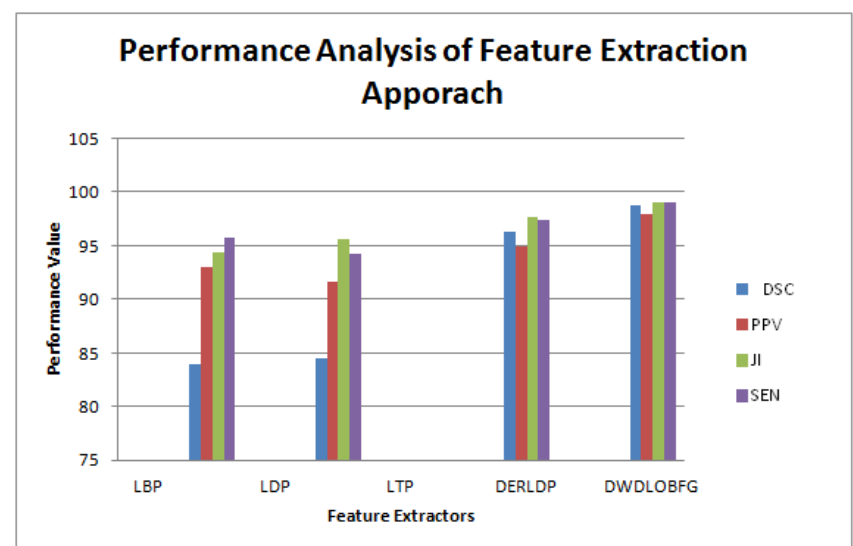

Fig.14 Examination of Feature Retrieval Approaches on BRATS 2015

From the examination of Figure 14, all performance measures values range 97-98 in DWDLOBFG. Because it provides superior values among other schemes. So the DWDLOBFG is most excellent for the proposed feature retrieval method.

Trial No 2: Examination of Brain Tumor Separating Approaches 
In this trial , this work will assess the proposed brain tumor separating approaches with altering kernel size.

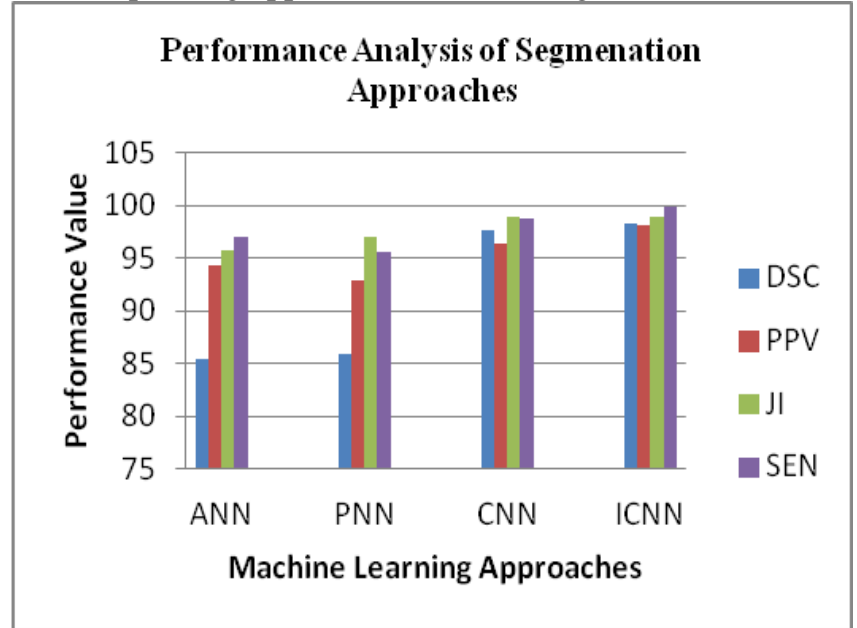

Figure. 15 Examination of Brain Tumor Separating Approaches on BRATS 2015

.From the examination of Figure 15, all performance measures values range 96-97 in ICNN. Because it provides superior values among other schemes. So the ICNN is most excellent for the proposed brain tumor separation scheme.

Trial No 3: Examination of Proposed Feature Retrieval Method through Different Kernel Range

In this trial , this work will assess the proposed feature retrieval schemes with altering kernel dimension.

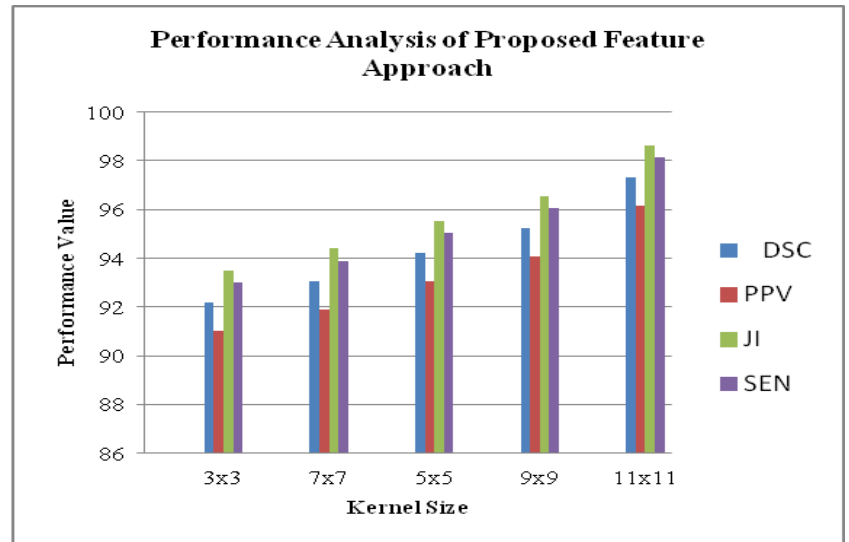

Fig.16 Examination of Feature Retrieving Approach by Various Kernel Size on BRATS 2015

From the examination of Figure.16, all performance measures values range 97-98 in 11x11 kernel. Because it provides superior values among other sizes. Thus the $11 \times 11$ kernel dimension is most excellent for the proposed feature system.

\section{CONCLUSION}

Separating brain tumors is a tedious job, as a result mistakes can guide to more. This paper provides an in-depth schema for extracting the characteristics of a localized classifier (DWDLOBFG) and an in-depth ICNN classification method for brain tumor separation. The test was performed on the BRATS 2015, the work proofed that DWDLOBFG using the ICNN method offers the most excellent outputs than other methods. In accordance with DICE and Jaccard, this work declares that the outputs of DWDLOBFG with the ICNN are related to this part of the reality. To enhance ICNN, this work intends to broaden the picture of gaining knowlege as well as develop effectual system for extracting features to improve ICNN.

\section{REFERENCES}

1. Abd-Ellah M. K., Awad A. I., Khalaf A. A. M., Hamed H. F. A. "Classification of brain tumor MRIs using a kernel support vector machine", Sprig, pp. 151-160, 2016.

2. Abd-Ellah M. K., Awad A. I., Khalaf A. A. M., Hamed H. F. A "Design and implementation of a computer-aided diagnosis system for brain tumor classification", IEE Inter. Conf. Microele., pp. 73-76, 2016.

3. Arakeri M. P., Reddy G. R. M., "Computer-aided diagnosis system for tissue characterization of brain tumor on magnetic resonance images", Signal, Image Video Processing, 9(2), 409-425,2015.

4. Browniee, J., "Overfitting and Underfitting With Machine Learning Algorithms"., March 21, 2016.

5. Casamitjana A., Puch S., Aduriz A., Vilaplana V., "Convolutional neural networks for brain tumor Separating : a comparison of multi-resolution architectures", Sprig, pp. 150-161, 2016.

6. Chang P. D., " Fully convolutional deep residual neural networks for brain tumor Separating". Sprig, pp. 108-118, 2016.

7. Cheng, J., Huang, W., Cao, S. "Classification via Tumor Region Augmentation and Partition". PLoS One, 10(10), 2015.

8. Da C., Zhang H., Sang Y.,"Brain CT image classification with deep neural networks", Sprig Inter. Pub., vol. 1, pp. 653-662, 2015.

9. Dandıl E., M. Çakıroglu, Z. Ek, "Computer-aided diagnosis of malign and benign brain tumors on MR images", Sprig, pp. 157-166,2015.

10. Deepa S. N., Devi B. A., "Artificial neural networks design for classification of brain tumour", IEEE, pp. 1-6, 2012.

11. El-Dahshan E.-S. A., Mohsen H. M., Revett K., Salem A.-B. M., "Computer-aided diagnosis of human brain tumor through MRI: A survey and a new algorithm", Expert Systems Applications. 41(11), 5526-5545,2014.

12. Gao X. W., Tian R. Hui, Z., "Classification of CT brain images based on deep learning networks", Computing Methods Programming Biomedical. 138, 49-56,2017.

13. Goswami S., Bhaiya L. K. P., " Brain tumor recognition using unsupervised learning based neural network", IEEE, pp. 573-577, 2013.

14. Havaei M., Davy A., Warde-Farley D., et al, " Brain tumor Separating with deep neural networks", Medical Image Analysis, 35, 18-31,2017.

15. He, K., Zhang, Ren X., et al, "Deep residual learning for Image Recognition", CVPR, 770-778, 2015.

16. Hemanth D. J., Vijila C. K. S., et al., " Performance improved iteration-free artificial neural networks for abnormal magnetic resonance brain image classification", Neural Computing. 98-107, 2014.

17. Hinton G. E., Osindero S., Teh Y.W., "A fast learning algorithm for deep belief nets". Neural Computing. 18(7), 1527-1554,2006.

18. Jayadevappa D., Srinivas Kumar S., Murty D., "Medical image Separating algorithms using deformable models: a review", IETE Technology. Revision. 28(3), 248-255,2011.

19. Jia Y. Xu, Z., Ai Y., et al., "Deep convolutional activation features for large scale brain tumor histopathology image classification and separating", IEEE, pp. 947-951,2015.

20. Jiang J., Trundle P., Ren J., "Medical image Examination with artificial neural networks", Computing Medical Imaging Graphics, 34(8), 617-631,2010.

21. Krizhevsky A., Sutskever I., Hinton G. E., "Classification with Deep Convolutional Neural Networks", pp. 1097-1105, 2012.

22. Lakshmi Devasena C., Hemalatha M., "Efficient computer aided diagnosis of abnormal parts recognition in magnetic resonance images using hybrid abnormality recognition algorithm", Journal of Computer Science. 3(3), 117-128,2013.

23. LeCun Y., Kavukcuoglu K., Farabet C., "Convolutional networks and applications in vision" , IEEE, pp. 253-256,2010.

24. Lei J., Li G., Zhang J., Guo Q., Tu D., "Continuous action Separating and recognition using hybrid convolutional neural network-hidden Markov model", IET Computing Vision. 10, 537-5447,2016.

25. Logeswari. T., Karnan M., "An improved implementation of brain tumor recognition using separating based on hierarchical self organizing map", International Journal of Computing Theory Engineering, 2(4), 591,2010.

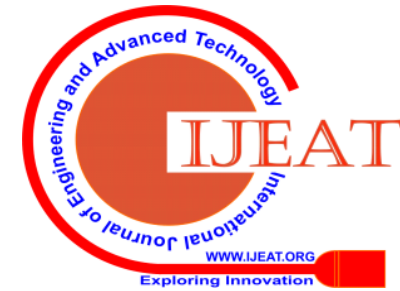


26. Madabhushi A., Lee G., "Image Examination and machine learning in digital pathology: challenges and opportunities", Medical Image Analysis. 33, 170-175, 2016.

27. Patil S., Udupi V. R., "A computer aided diagnostic system for classification of brain tumors using texture features and probabilistic neural network", International. Journal of Computer Science Engineering Information Technology 3, 61-66, 2013.

28. Pereira S., Oliveira A., Alves V., Silva C. A., " On hierarchical brain tumor Separating in MRI using fully convolutional neural networks: a preliminary study", pp,1-4, 2017.

29. Pereira S., Pinto A., Alves V., Silva C. A., "Brain tumor Separating using convolutional neural networks in MRI images",. IEEE Trans. Med. Img. 35(5), 1240-1251, 2016.

30. Senta A., et al, "Performance evaluation of support vector machine and convolutional neural network algorithms in real-time vehicle type classification", Sprig., pp. 934-943,2018.

31. Suk H., et al. "Deep ensemble learning of sparse regression models for brain disease diagnosis", Medical Image Analysis. 37, 101-113, 2017.

32. Yan C., et al, "Driving posture recognition by convolutional neural networks". IET Computer Vision 10, 103-11411,2016.

33. Yan C., et al, "Supervised hash coding with deep neural network for environment perception of intelligent vehicles", IEEE Transactions on Intelligent Transport Systems. 19(1), 284-295,2018.

34. Yazdani S., et al, "Image Separating methods and applications in MRI brain images", IETE Techologies, 32(6), 413-427, 2015.

35. Zhao L., Jia K., "Multiscale CNNs for brain tumor Separating and diagnosis", Computer Mathematical Methods, 2016.

36. Zhao X., et al, "Brain tumor Separating using a fully convolutiona neural network with conditional random fields", Sprig, pp. 75-87, 2016.

\section{AUTHHORS PROFILE}

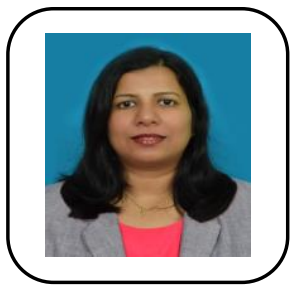

Ms. Nisha Joseph is a Computer Engineer working as Assistant Professor in Computing at SAITGITS College of Engineering, Kerala, India since 2004. She has been in to teaching for more than a decade. She is a Research Scholar in Manaonmaniam Sundaranar University, TN, India and her research is focused on Medical Imaging thrusting Brain Tumors. Her research interests include Digital Image Processing, Big data and Computer networking. She did B.Tech in Computer Engineering at Cochin University of Science and Technology and M.Tech in Computer and Information Technology at Manonmaniam Sundaranar University.

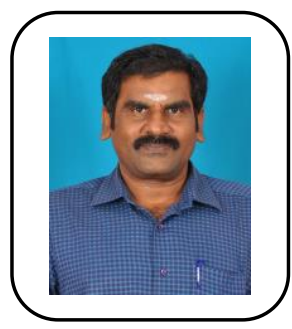

Dr. D Murugan is a Professor at Manonmaniam Sundaranar University, India. He received a bachelor degree in Electronic and Communication from Madurai Kamaraj University and master's degree in Computer Science and Engineering from Madurai Kamaraj University, India. He Completed his Ph.D in Computer Science and Engineering from M.S University, India. His area of expertise is Face Recognization. He is interested in Image Processing and Software Quality Engineering.

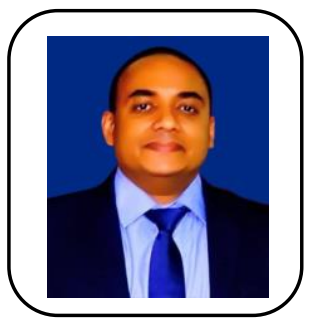

Dr. Basil John Thomas is a Chartered Management Consultant and Fellow from CMC Canada. He currently holds and serves the position of Assistant Professor in Human Resources at Sur University College affiliated to Bond University Australia and the University of Sunderland, UK. He is the recipient of the GCC Educational Leadership Award for the 'Best Professor in HR and Organizational Studies' honored by the World Federation of Academic \& Educational Institutions Jury in 2019 March. Later he was honored with various citations including the Global Outreach Research and education Award in 2019 April for Outstanding teacher in Management and the Hundred most talented professors in the world by the World Educational Congress 2019. In July 2019 he was honored with Oman Education Leadership Award and recently the Asian Education Leadership Award in October 2019 for the Best Professor in Finance.

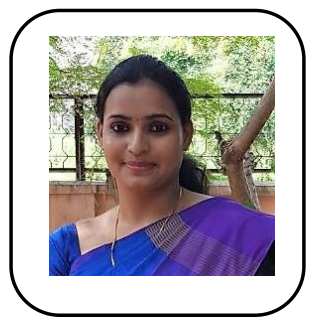

Dr. A Ramya is working as a Assistant Professor in the department of Computer Applications, B.S. Abdur Rahman Crescent Institute of Science and Technology, Chenai, India. She has received the doctoral degree in Computer Science from Manonmaniam Sundaranar University, Tirunelveli, India. She has completed the Masters in Computer Science from Lady College, Madurai, India. Remote sensing and Machine Learning algorithms. 